\section{Inflammatory Esophageal Polyp with Pseudosarcomatous Lesion}

A 73-year-old woman was admitted due to dysphagia. A polyp in the lower esophagus was detected by endoscopic and barium examinations. Endoscopy demonstrated a small polypoid lesion with a stalk about $10 \mathrm{~mm}$ in diameter, and revealed reflux esophagitis (Figure 1). A biopsy of the tumor (Figure 2) suggested malignancy, with suspected poorly-differentiated squamous-cell carcinoma or carcinosarcoma. Since malignancy could not be excluded (1), a polypectomy was performed for diagnostic purposes. The histological findings revealed erosion, squamous epithelial hyperplasia, and numerous bizarre atypical spindle cells concentrating underneath the exudative cap and tapering off into the adjacent granulation tissue. Although the nuclei of the atypical cells were pleomorphic and hyperchromatic, few mitotic figures were observed. These bizarre cells displayed positive staining for vimentin and negative staining for keratin, desmin, and epithelial membrane antigen (EMA). Based on these findings, the histological diagnosis was an inflammatory polyp of the esophagus with a pseudosarcomatous lesion. The patient is now well and no polyp was found 12 months after the polypectomy.

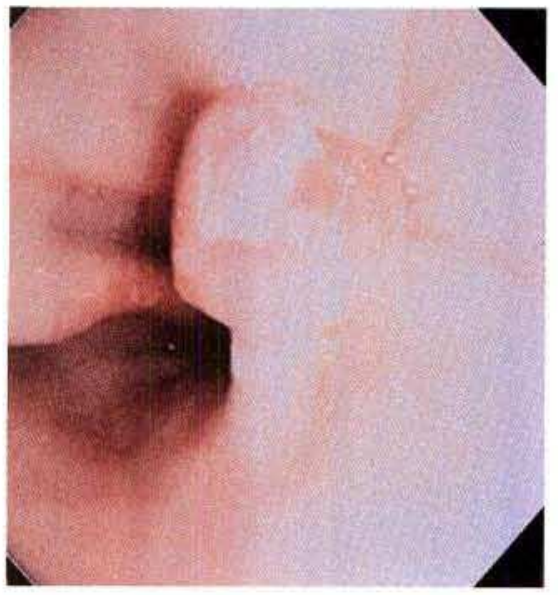

Figure 1: Endoscopic view of the esophageal polyp with stalk.

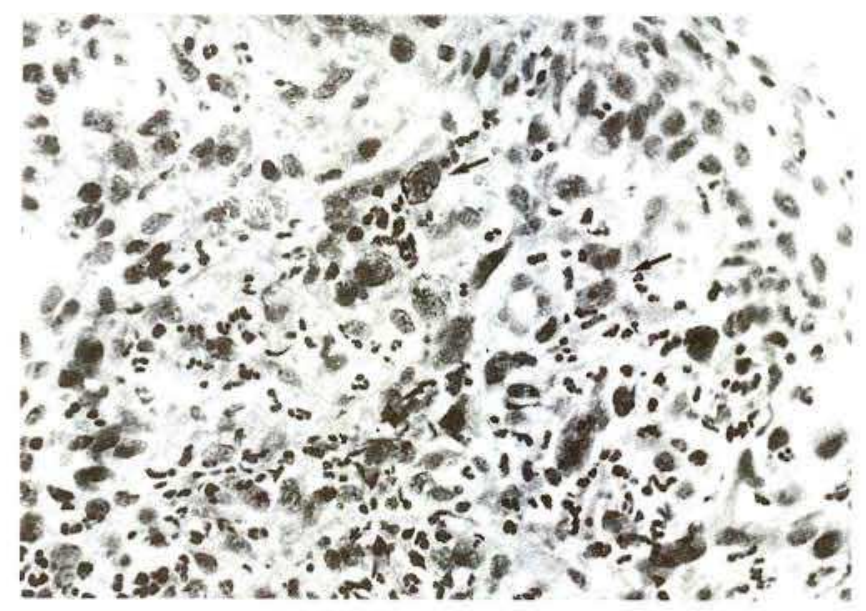

Figure 2: Histological findings in the biopsy specimen. Numerous atypical stromal cells (arrows) are seen in granulation tissue.
Esophageal inflammatory polyps with pseudosarcomatous lesions are uncommon, and often occur near the esophagogastric junction (2). They are almost always less than $20 \mathrm{~mm}$ in size (3), while pseudosarcomatous or true carcinosarcomatous polyps are over $50 \mathrm{~mm}$ (4). These pseudosarcomatous lesions are characterized by atypical proliferation of mesenchymal cells that are considered to be fibroblastic or myofibroblastic in origin (3), and the immunohistochemical results in this case were consistent with a mesenchymal derivation. Pseudosarcomatous changes are seen in esophageal ulcerative lesions caused by reflux esophagitis. However, differential diagnosis between pseudosarcomatous change and malignancy is rather difficult in polypoid lesions, as their clinical appearances are nearly identical. Eighteen percent of inflammatory tumors with bizarre stromal cells were reported to be diagnosed as malignant (3). Correct recognition of such pseudosarcomatous lesions is clinically important, since they never progress to malignancy. We would therefore suggest that it is worthwhile to obtain a polypectomized specimen to minimize the diagnostic pitfalls of pathological examination and avoid unnecessary surgery.

\section{T. Ajiki', T. Fujimori', Y. Hamabe', Y. Saitoh', N. Aoyama',}

T. Nakamura', S. Maeda', K. Nagasakot

'First Dept. of Surgery. Kobe University School of Medicine.

Kobe, Japan

${ }^{2}$ Second Dept. of Pathology, Kobe University School of Medicine, Kobe, Japan

${ }^{3}$ Second Dept. of Internal Medicine, Kobe University School of Medicine, Kobe, Japan

+ Gunma Cancer Center Institute, Ota, Japan

\section{References}

1. Kuhajda FP, Sun T, Mendelsohn G. Polypoid squamous carcinoma of the esophagus: a case report with immunostaining for keratin. Am J Surg Pathol 1983; 7: 495-9.

2. Wolf BC, Khettry U, Leonardi HK, et al. Benign lesions mimicking malignant tumors of the esophagus. Hum Pathol 1988; 19: 148-54

3. Shekitka KM, Helwig EB. Deceptive bizarre stromal cells in polyps and ulcers of the gastrointestinal tract. Cancer 1991; 67: $2111-7$.

4. Matsusaka T, Watanabe H, Enjoji M. Pseudosarcoma and carcinosarcoma of the esophagus, Cancer 1976; 37: 1546-55.

\section{Corresponding Author}

T. Ajiki, M.D.

First Department of Surgery

Kobe University School of Medicine

7-5-2 Kusunoki-cho, Chuo-ku, Kobe

Hyogo, 650

Japan

Fax: $+81-78-382-2110$ 